\title{
Radiotherapy as salvage treatment of salivary duct carcinoma in major salivary glands without radical operations
}

This article was published in the following Dove Press journal:

Cancer Management and Research

\author{
Lu $\mathrm{Di}^{1-3, *}$ \\ Kai Qian ${ }^{2,4, *}$ \\ Chengrun $\mathrm{Du}^{1,2}$ \\ Chunying Shen ${ }^{1,2}$ \\ Ruiping Zhai ${ }^{1,2}$ \\ Xiayun $\mathrm{He}^{1,2}$ \\ Xiaoshen Wang ${ }^{1,2}$ \\ Tingting $\mathrm{Xu}^{1,2}$ \\ Chaosu $\mathrm{Hu}^{1,2}$ \\ Hongmei Ying ${ }^{1,2}$ \\ 'Department of Radiation Oncology, \\ Fudan University Shanghai Cancer \\ Center, Shanghai 200032, China; \\ ${ }^{2}$ Department of Oncology, Shanghai \\ Medical College, Fudan University, \\ Shanghai 200032, China; ${ }^{3}$ Department \\ of Oncology, Liyang People's Hospital, \\ Changzhou, Jiangsu 213000, China; \\ ${ }^{4}$ Department of Head and Neck \\ Surgery, Fudan University Shanghai \\ Cancer Center, Shanghai 200032, \\ China \\ *These authors contributed equally to \\ this work
}

Correspondence: Hongmei Ying Department of Radiation Oncology, Fudan University Shanghai Cancer Center, 270 Dong an Road, Shanghai 200032, China

$\mathrm{Tel}+862164175590$

Fax +862164174774

Email doctoryinghm@163.com
Background: Salivary duct carcinoma (SDC) is an extremely rare and highly malignant carcinoma, and surgical radical resection is the most effective therapy. However, there were quite a proportion of patients receiving non-radical resections, and how to treat them remained controversial. Thus, the aim of this study is to evaluate whether postoperative radiotherapy could be a salvage treatment of SDC in major salivary glands without radical operations.

Patients and methods: We identified 40 pathologically diagnosed SDC patients who came to our hospital and did not receive radical operations. Thirty-three patients received at least one treatment (remedial operation, postoperative radiotherapy and chemotherapy), and seven patients only chose observation and received no further treatment. The prognostic indicators of the local-regional control (LRC) and distant disease-free survival were analyzed using the Kaplan-Meier methods and the Cox proportional hazards regression models.

Results: Thirteen patients experienced local-regional recurrence or local progression, and distant metastases were observed in 15 patients. Through multivariate analysis, we found that postoperative radiotherapy was associated with better LRC, but this kind of treatment did not show significant efficacy in the prevention of distant metastasis.

Conclusion: SDC is a rare, aggressive malignancy, and a substantial proportion of these patients experienced inadequate initial treatments. Although postoperative radiotherapy could not decrease distant metastases, it might help to improve LRC in patients with SDC.

Keywords: salivary duct carcinoma, prognosis, non-radical resection, postoperative radiotherapy

\section{Introduction}

Salivary duct carcinoma (SDC) is a rare malignancy and has relatively aggressive features among salivary cancers, which was first described by Kleinsasser et al ${ }^{1}$ in 1968. The tumor arises from the ductal epithelium of the salivary gland, and the incidence was reported to be varying between $1 \%$ and $3 \%$ of all the malignant salivary tumors. ${ }^{2}$ Because of similarity in cell morphology and expression of androgen receptor and HER-2, SDC was considered histologically similar to ductal carcinoma of the breast. However, there is still a lack of effective systemic therapy of this disease. Nowadays, primary treatment consisting of wide local resection of the primary lesion especially a total parotidectomy and neck dissection is considered necessary and strongly recommended for SDC. ${ }^{3,4}$ Nevertheless, there is still quite a proportion of these patients receiving non-radical resections because both the preoperative diagnosis and the radical resection are quite difficult. Postoperative adjuvant radiotherapy was proved effective and advised for salivary gland cancers with aggressive features. ${ }^{5-7}$ However, the role 
of postoperative radiation therapy in SDC without radical operations remains controversial. Therefore, the purpose of this study is to evaluate whether postoperative radiotherapy could be a salvage treatment of SDC in major salivary glands without radical operations.

\section{Patients and methods}

\section{Patients}

From August 2008 to March 2017, a total of 40 patients pathologically diagnosed with SDC who came to our hospital and did not receive radical operations were included and reviewed in this study. The patients accepted initial operations in different hospitals, and the extent of surgery for them was all inadequate in the region of salivary glands or cervical lymph nodes. Twenty-two cases did not have adequate surgical margins of the primary tumors and most of them just received mass excisions. Neck dissection was only performed in 10 of the 40 patients. In addition, 14 patients had gross residual diseases because of the extended tumor invasion or non-standard operation. The clinicopathological data, therapeutic procedures and clinical outcomes were retrospectively reviewed. Patients with distant metastasis at initial treatment (cM1) were excluded from the study. The study was reviewed and approved by the institutional ethics committee of Fudan University Shanghai Cancer Center, and all participants or their guardians gave written informed consent.

\section{Treatment}

When these patients came to hospital, complementary therapies including operation and radiotherapy were routinely recommended. Finally, 33 patients received at least one treatment (remedial operation, postoperative radiotherapy and chemotherapy); 7 patients only chose observation and received no further treatment (Table 1). Only four patients underwent reoperations. There were two main reasons. First, most of the patients did not have gross residual diseases. Considering the surgical complications and value of re-operation, reoperations should be cautious. Second, for patients with gross residual disease, secondary resection was very difficult and risky.

Intensity-modulated radiotherapy was used for adjuvant radiotherapy. For radiotherapy, gross tumor volume was defined as the remaining primary lesions and the positive cervical lymph nodes, and clinic target volume (CTV) was defined as tumor bed, subclinical lesion and possibly invaded area. Taking some systematic errors into consideration, the margin of the planning target volume was $5 \mathrm{~mm}$ beyond the
Table I Clinicopathological characteristics of all the included patients

\begin{tabular}{|l|l|}
\hline Clinical characteristics & Result \\
\hline Age, average (range), years & $58.5(39-79)$ \\
Sex, $\mathrm{n}(\%)$ & \\
$\quad$ Female & $5(12.5)$ \\
Male & $35(87.5)$ \\
Smoking history, $\mathrm{n}$ (\%) & \\
$\quad$ Negative & $1 \mathrm{I}(27.5)$ \\
Positive & $29(72.5)$ \\
Preoperative symptoms, $\mathrm{n}(\%)$ & \\
No & $28(70)$ \\
Yes & $1 \mathrm{I}(27.5)$ \\
Unknown & $1(2.5)$ \\
Primary tumor site, $\mathrm{n}$ (\%) & \\
Submandibular gland & $9(22.5)$ \\
Parotid gland & $31(77.5)$ \\
Tumor size, average (range), mm, $\mathrm{n}(\%)$ & $27.5(10-65)$ \\
$\leq 25$ & $22(55.0)$ \\
$>25$ & $12(30.0)$ \\
Unknown & $6(15.0)$ \\
Gross residual disease (primary or lymph node), $\mathrm{n}(\%)$ & \\
No & $26(65.0)$ \\
Yes & $14(35.0)$ \\
Adequate surgical margins, $\mathrm{n}$ (\%) & \\
No & $22(55.0)$ \\
Yes & $18(45.0)$ \\
Neck dissection, $\mathrm{n}$ (\%) & \\
No & $30(75.0)$ \\
Yes & $10(25.0)$ \\
Treatment, $\mathrm{n}$ (\%) & \\
Follow-up & $7(17.5)$ \\
Operation & $\mathrm{I}(2.5)$ \\
Chemotherapy & $2(5.0)$ \\
Radiotherapy & $24(67.5)$ \\
Radiotherapy + chemotherapy & $3(7.5)$ \\
Operation + radiotherapy & $2(5.0)$ \\
Operation + radiotherapy + chemotherapy & $1(2.5)$ \\
\hline
\end{tabular}

CTV. The median prescribed dose of these patients ranged from 54 to $70 \mathrm{~Gy}$.

Chemotherapy was not routinely performed on SDC patients. There were several regimens of adjuvant chemotherapy in our institution, including GP scheme (gemcitabine + cisplatin) and TP scheme (paclitaxel + cisplatin).

\section{Follow-up}

After the completion of treatments, patients were followed up every 3 months in the first year, then every 3-6 months for the next 4 years and yearly thereafter. All patients were followed up until the event of death or until the time of analysis. Local conditions of the patients were evaluated through physical examination, neck ultrasound and imaging examinations of the head and neck regions. Chest computed 
tomography (CT) and abdominal sonography were performed routinely to exclude distant metastasis. Aspiration biopsy, positron-emission tomography and isotope bone scan were carried out if indicated.

\section{Statistical analyses}

The duration of time to locoregional failure and distant metastasis was calculated from the end of the remedial treatment to the event occurrence. The probability of the local-regional control (LRC) and distant disease-free survival (DDFS) was calculated by the Kaplan-Meier methods and the Cox proportional hazards models. A stepwise forward method was used, and the likelihood ratio tests were carried out to identify significant independent predictors. HR parameters were determined using the Wald tests. $P$-values $<0.05$ were considered statistically significant. The SPSS 22.0 software was used to analyze the statistics.

\section{Results}

\section{Patients' characteristics}

The clinical characteristics of the 40 patients are summarized in Table 1 . The vast majority of them are males, and only five patients are females. The age of the patients ranges from 39 to 79 years, with a median age of 58.5 years. Eleven patients had preoperative symptoms including pain, facial paralysis and numbness. Among all of them, 14 patients were found with residual primary lesions or residual lymph nodes through postoperative evaluations (ultrasound, CT or magnetic resonance image). After treatment completion, the median follow-up time was 29 months, with a range from 8 to 119 months.

\section{Local-regional control}

During the follow-up period, 13 patients experienced localregional recurrence or local progression. Six cases occurred at the location of salivary gland with the remaining seven occurring in the cervical lymph nodes. The Kaplan-Meier curve indicates that patients receiving postoperative radiotherapy had better LRC than those without radiotherapy $(P=0.000)$ (Figure 1). Radiotherapy showed good effects especially in the first 40 months. In the multivariate analysis using a Cox proportional hazard model, gross residual disease $(\mathrm{HR}=9.499$, $P=0.047)$ and no postoperative radiotherapy $(\mathrm{HR}=70.567$, $P=0.010)$ are both significant risk factors for LRC (Table 2).

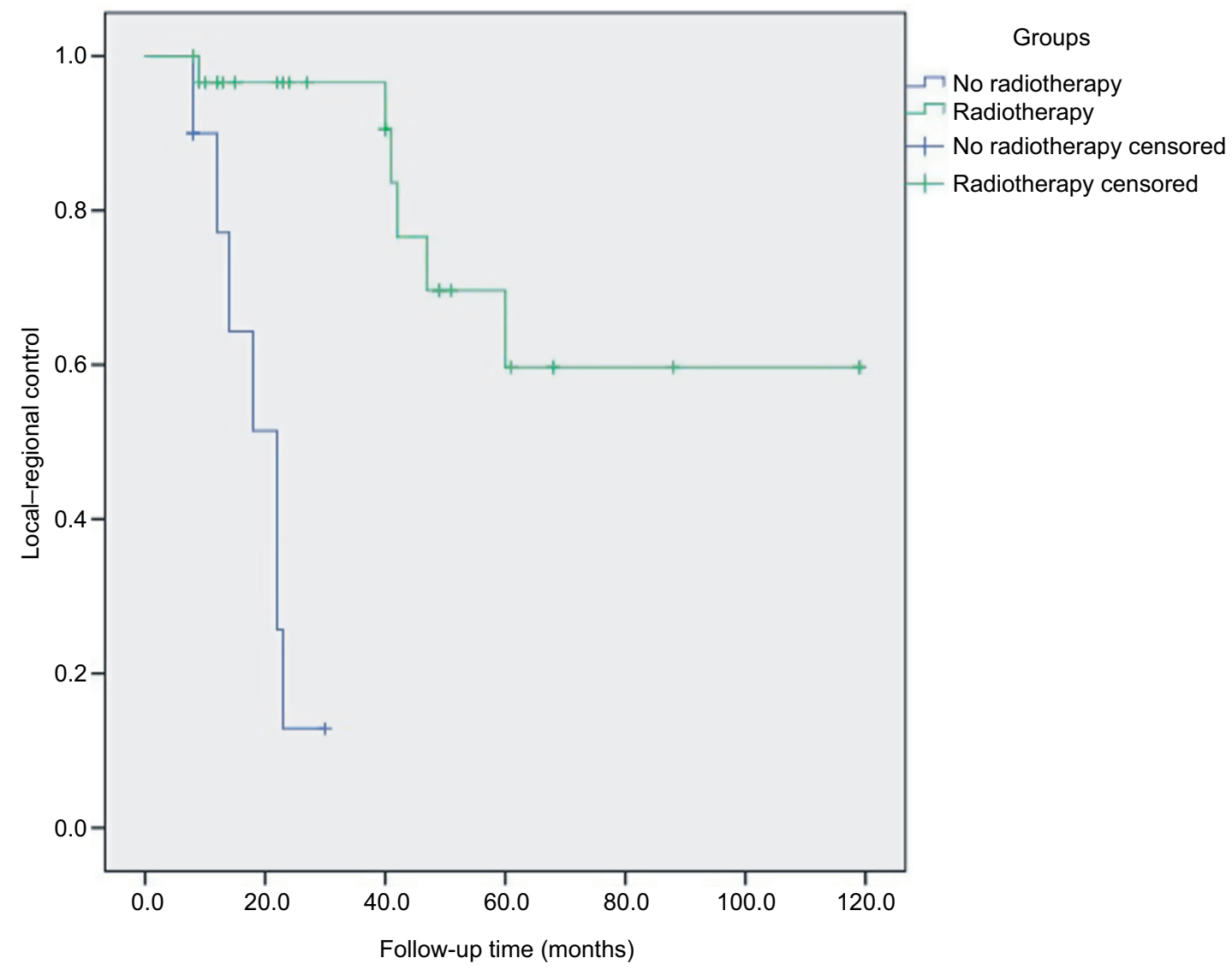

Figure I Kaplan-Meier analysis of local-regional control of patients with/without radiotherapy (Log-rank tests: $\chi^{2}=25.394, P=0.000$ ). 
Table 2 Univariate and multivariable analyses for LRC

\begin{tabular}{|c|c|c|c|c|}
\hline \multirow[t]{3}{*}{ Variables } & \multicolumn{4}{|l|}{ LRC } \\
\hline & \multicolumn{2}{|c|}{ Univariate analysis } & \multicolumn{2}{|l|}{ Multivariate analysis } \\
\hline & $\chi^{2}$ test & $P$-value & HR (95\% Cl) & $P$-value \\
\hline Age, years & 6.171 & 0.013 & & 0.077 \\
\hline$\leq 50$ & & & I & \\
\hline$>50$ & & & II.I3I (0.769-I6I.204) & \\
\hline Gender & 0.002 & 0.960 & & 0.470 \\
\hline Female & & & 1 & \\
\hline Male & & & $3.037(0.149-61.826)$ & \\
\hline Smoking history & 0.154 & 0.694 & & 0.153 \\
\hline No & & & I & \\
\hline Yes & & & $3.675(0.616-21.926)$ & \\
\hline Tumor size, mm & 0.094 & 0.760 & & 0.080 \\
\hline$\leq 25$ & & & 1 & \\
\hline$>25$ & & & $0.104(0.008-1.313)$ & \\
\hline Gross residual disease & 5.303 & 0.021 & & 0.047 \\
\hline No & & & I & \\
\hline Yes & & & $9.499(1.032-87.406)$ & \\
\hline Radiotherapy & 25.394 & 0.000 & & 0.010 \\
\hline Yes & & & I & \\
\hline No & & & $70.567(2.7 \mid 9-1,831.238)$ & \\
\hline Remedial surgery & 1.930 & 0.165 & & 0.839 \\
\hline Yes & & & I & \\
\hline No & & & $0.703(0.023-21.201)$ & \\
\hline
\end{tabular}

Notes: Bold figures indicate statistical significant, $P<0.05$.

Abbreviation: LRC, local-regional control.

\section{Distant disease-free survival}

Prone to metastasis was considered as the main reason for the failure of therapy and the death of patients. The DDFS rate for these patients in 5 years was $56.8 \%$. Distant metastases were observed in 15 patients, and the most common location of the metastasis was lungs $(9 / 15)$. In univariate analysis, gross residual disease $\left(\chi^{2}=1.911, P=0.001\right)$ and receiving remedial surgery $\left(\chi^{2}=7.283, P=0.007\right)$ are risk factors. But the multivariate analysis shows that smoking history (HR $=13.694, P=0.024)$ and gross residual disease $(\mathrm{HR}=2.747, P=0.029)$ are associated with distant metastasis. Postoperative radiotherapy did not decrease the incidence of distant metastasis from the abovementioned results (Figure 2 and Table 3).

\section{Patients with gross residual disease}

Twelve patients suffered from residual of primary lesions, and two patients had positive lymph nodes after initial operations. Among them, eleven patients received further local therapy including surgery and radiotherapy. On the other hand, there were still three patients receiving no operation and radiotherapy, and one of them accepted chemotherapy. Detailed conditions of patients are shown in Table 4. Six patients suffered from local-regional progressive disease, and ten cases had distant metastases during the follow-up period. Most patients undergoing radiotherapy responded well to the treatment and had better local control.

\section{Discussion}

SDC is one of the most aggressive salivary gland carcinomas. ${ }^{8,9}$ The disease has a high propensity for lymph node metastasis and local aggression. ${ }^{10}$ In order to improve the success rate of the treatment of SDC, accurate preoperative diagnosis and stage, normative surgery practice and necessary adjuvant treatment are needed. However, the lack of accurate pathologic diagnosis of SDC before or during the operation and enough cognition to this rare disease leads to a substantial proportion of these patients not receiving standardized operations at initial diagnosis. In addition, there were also some patients receiving non-radical resections because of extensive tumor invasion. No consensus has been reached on the subsequent treatment for this subset of patients because very little was found in the literature on this question. Therefore, our study is to analyze retrospectively the patients without radical operations so as to investigate whether postoperative radiation would improve outcomes. 


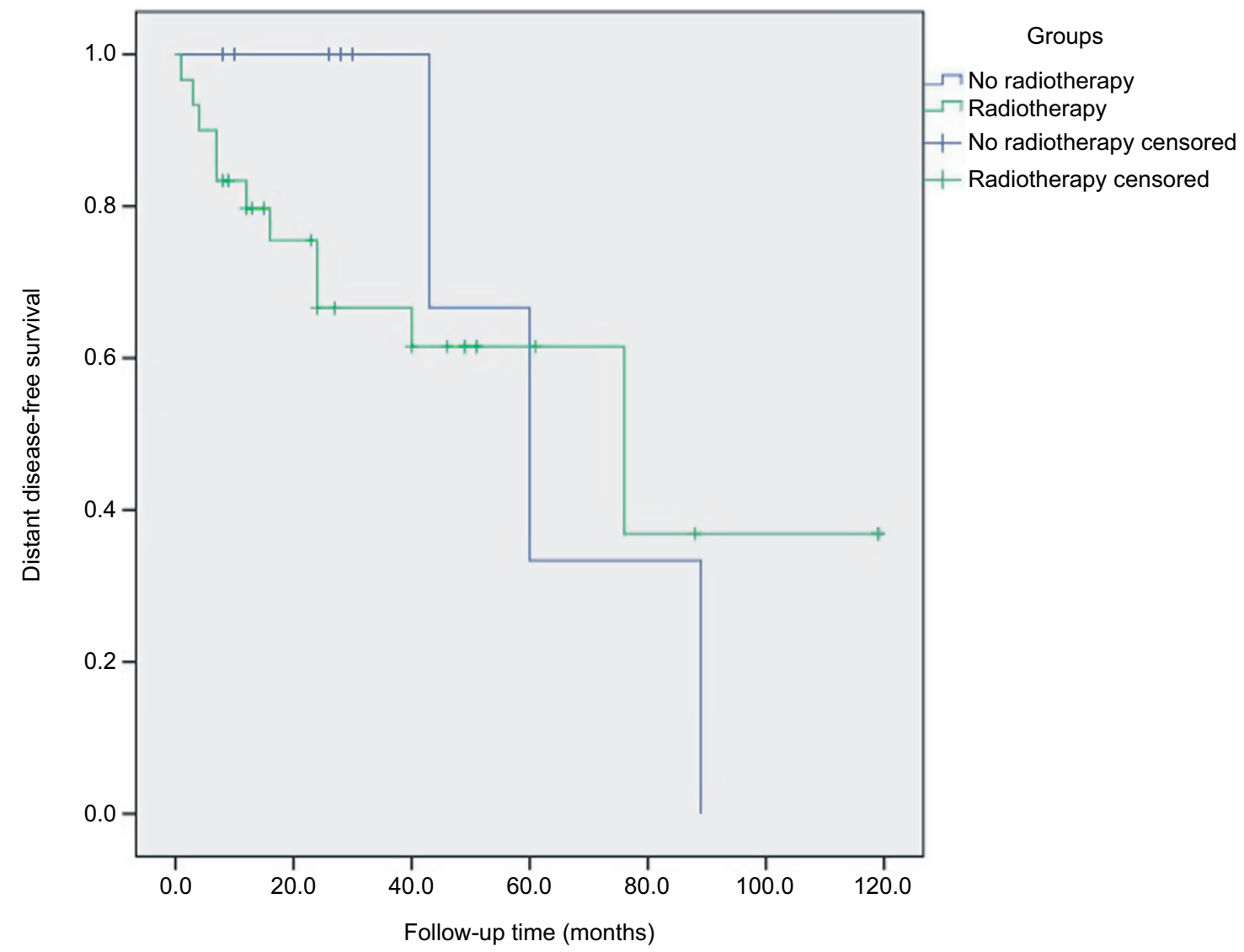

Figure 2 Kaplan-Meier analysis of distant disease-free survival of patients with/without radiotherapy (Log-rank tests: $\left.\chi^{2}=0.191, P=0.662\right)$.

Table 3 Univariate and multivariable analyses for DDFS

\begin{tabular}{|c|c|c|c|c|}
\hline \multirow[t]{3}{*}{ Variables } & \multicolumn{4}{|l|}{ DDFS } \\
\hline & \multicolumn{2}{|c|}{ Univariate analysis } & \multicolumn{2}{|l|}{ Multivariate analysis } \\
\hline & $\chi^{2}$ test & $P$-value & HR (95\% Cl) & $P$-value \\
\hline Age, years & 1.124 & 0.289 & & 0.918 \\
\hline$\leq 50$ & & & 1 & \\
\hline$>50$ & & & $0.912(0.155-5.350)$ & \\
\hline Gender & 0.181 & 0.670 & & 0.098 \\
\hline Female & & & 1 & \\
\hline Male & & & $0.113(0.009-1.500)$ & \\
\hline Smoking history & 1.366 & 0.243 & & 0.024 \\
\hline No & & & 1 & \\
\hline Yes & & & $13.694(1.4 \mid 2-132.798)$ & \\
\hline Tumor size, mm & 1.750 & 0.186 & & 0.186 \\
\hline$\leq 25$ & & & 1 & \\
\hline$>25$ & & & $2.747(0.615-12.279)$ & \\
\hline Gross residual disease & 10.911 & 0.001 & & 0.029 \\
\hline No & & & 1 & \\
\hline Yes & & & $10.592(1.270-88.346)$ & \\
\hline Radiotherapy & 0.191 & 0.662 & & 0.367 \\
\hline Yes & & & 1 & \\
\hline No & & & $0.44 I(0.074-2.610)$ & \\
\hline Remedial surgery & 7.283 & 0.007 & & 0.050 \\
\hline Yes & & & 1 & \\
\hline No & & & $0.065(0.004-1.000)$ & \\
\hline
\end{tabular}

Notes: Bold figures indicate statistical significant, $P<0.05$.

Abbreviation: DDFS, distant disease-free survival. 
Table 4 Treatment and prognosis of patients with gross residual disease

\begin{tabular}{|l|l|l|l|l|l|}
\hline & Treatment & $\begin{array}{l}\text { Local-regional } \\
\text { progression }\end{array}$ & $\begin{array}{l}\text { Time } \\
\text { (months) }\end{array}$ & $\begin{array}{l}\text { Distant } \\
\text { metastasis }\end{array}$ & $\begin{array}{l}\text { Time } \\
\text { (months) }\end{array}$ \\
\hline Patient I & Radiotherapy & No & 88 & No & 88 \\
Patient 2 & Radiotherapy & No & 12 & Yes & 7 \\
Patient 3 & Chemotherapy & Yes & 18 & Yes & Yes \\
Patient 4 & Radiotherapy & No & 22 & Yes & 7 \\
Patient 5 & Radiotherapy + chemotherapy & No & 15 & No & 12 \\
Patient 6 & Radiotherapy & Yes & 9 & Yes & 13 \\
Patient 7 & Radiotherapy & No & 10 & Yes & 16 \\
Patient 8 & Operation + radiotherapy & Yes & 40 & Yes & 40 \\
Patient 9 & Operation + radiotherapy & No & 40 & No & 10 \\
Patient 10 & Operation & Yes & 8 & Yes \\
Patient II & Operation + radiotherapy + chemotherapy & No & 12 & Yes & 4 \\
Patient I2 & Follow-up & Yes & 12 & Yes \\
Patient I3 & Radiotherapy & No & 13 & No \\
Patient 14 & Follow-up & Yes & 23 & 1 \\
\hline
\end{tabular}

Based on our results, we demonstrated the benefit of radiotherapy on LRC. However, this kind of treatment did not show significant efficacy in the prevention of distant metastasis.

Inadequate initial treatments and gross residual disease result in poor prognosis in patients with salivary gland cancer. A previous study analyzed retrospectively their patients with incompletely treated major salivary gland tumors and found that failure to achieve gross total resection during initial surgery resulted in worse overall survival. ${ }^{11}$ Moreover, gross residual disease was an independent predictor in these cases. Similarly, multivariate analyses of both LRC and DDFS showed that gross residual disease was an important risk factor in this study. The patients are more likely to experience distant metastasis in the follow-up time. Therefore, how to diagnose residual lesion early influences the patients' prognosis. Imaging examination combined with fine needle aspiration is helpful in determining residual focus, particularly the positive lymph nodes. Lee et al ${ }^{12}$ also found that positron emission tomography/CT was more effective than CT for detecting recurrences of salivary gland malignances, and it may also be helpful in postoperative assessment.

Radiation therapy plays an important role in the treatment of SDC. For patients who undergo radical surgery, positive radiation therapy is recommended routinely. Roh et $\mathrm{al}^{13}$ reported that postoperative radiotherapy was one of the most important prognostic factors of overall survival compared to surgery only. Previous studies also showed that aggressive treatment through radical resection and postoperative locoregional radiotherapy might lead to more successful LRC. ${ }^{5,14}$ Remarkably, Shinoto et al ${ }^{15}$ and Al-Qahtani et $\mathrm{al}^{16}$ reported that most of the locoregional recurrences actually occurred along the mandibular nerve, the facial nerve or in the base of skull, which were beyond the radiation field. They suggested that expansion of irradiation field could be beneficial for some patients and further reduce the relapse rate. On the other hand, for patients whose disease is unresectable or who refuse surgery are typically managed with radiation alone, the effectiveness of treatment is not satisfactory. The 10-year survival rate in a heterogeneous population of patients with inoperable or unresectable salivary gland malignancies treated with radiation alone was only $15 \%-25 \%,{ }^{17}$ which is far lower than the rate of patients who experienced operations. Although to our knowledge there is no report in the English literature concerning the radiation after operation without radical resection, this study fills the bill and indicates the importance of postoperative radiation in these patients.

Distant metastasis is considered as the main reason for the failure of therapy and the death of patients, which mainly occurs among cases with gross residual disease. Multivariate analysis showed that radiotherapy did not reduce the incidence of distant metastasis. Therefore, alternative treatments are needed. Systemic chemotherapy was not routinely performed among the 40 patients in this study because there was no clear evidence of benefit from chemotherapy. The regimens based on platinum, taxane or gemcitabine had been used for recurrent or metastatic salivary gland carcinomas including SDC in previous studies. ${ }^{18}$ However, SDC showed poor response to chemotherapy, and the addition of chemotherapy did not improve distant control or overall survival. ${ }^{19-21}$ Thus, there has been no consensus regarding the role of chemotherapy. Moreover, recent data have demonstrated the 
potential effects of HER-2-targeting therapy and androgen deprivation therapy in improving DFS and OS rates, ${ }^{22-25}$ and in-depth research on the molecular biology of SDC will discover new therapeutic targets for the aggressive disease.

There are several limitations to the present study. First, it is a retrospective study, and the sample size is still small due to the rarity of SDC. Therefore, the selection bias cannot be ignored. Second, the applications of different treatment methods were not unified. For example, the dosages of radiotherapy and the chemotherapy regimens were different for these patients. Third, these patients experienced initial treatments in different hospitals, and the differences of medical diagnosis and treatment among regions and medical institutions could influence the effects of first surgery.

\section{Conclusion}

SDC is a rare, aggressive malignancy, and a substantial proportion of these patients experienced inadequate initial treatments. Incomplete initial surgery and gross residual diseases result in poorer disease outcome. Although postoperative radiotherapy could not decrease distant metastases, it might help to improve LRC in patients with SDC. Molecular biology-based systematic therapy deserves further study.

\section{Disclosure}

The authors report no conflicts of interest in this work.

\section{References}

1. Kleinsasser O, Klein HJ, Hübner G. [Salivary duct carcinoma. A group of salivary gland tumors analogous to mammary duct carcinoma]. Arch Klin Exp Ohren Nasen Kehlkopfheilkd. 1968;192(1): 100-105.

2. Hosal AS, Fan C, Barnes L, Myers EN. Salivary duct carcinoma. Curr Opin Otolaryngol Head Neck Surg. 2003;129(6):720-725.

3. Sood S, Mcgurk M, Vaz F. Management of salivary gland tumours: United Kingdom National Multidisciplinary Guidelines. J Laryngol Otol. 2016;130(S2):S142-S149.

4. Ali S, Palmer FL, Dilorenzo M, Shah JP, Patel SG, Ganly I. Treatment of the neck in carcinoma of the parotid gland. Ann Surg Oncol. 2014;21(9):3042-3048.

5. Qian K, di L, Guo K, Zheng X, Ji Q, Wang Z. Cervical lymph node metastatic status and adjuvant therapy predict the prognosis of salivary duct carcinoma. J Oral Maxillofac Surg. 2018;76(7):1578-1586.

6. Terhaard CH, Lubsen $\mathrm{H}$, Rasch CR, et al. The role of radiotherapy in the treatment of malignant salivary gland tumors. Int J Radiat Oncol Biol Phys. 2005;61(1):103-111.

7. Kaur J, Goyal S, Muzumder S, Bhasker S, Mohanti BK, Rath GK. Outcome of surgery and post-operative radiotherapy for major salivary gland carcinoma: ten year experience from a single institute. Asian Pac J Cancer Prev. 2014;15(19):8259-8263.
8. Gilbert MR, Sharma A, Schmitt NC, et al. A 20-year review of 75 cases of salivary duct carcinoma. JAMA Otolaryngol Head Neck Surg. 2016;142(5):489-495.

9. Boon E, Bel M, van Boxtel W, et al. A clinicopathological study and prognostic factor analysis of 177 salivary duct carcinoma patients from The Netherlands. Int J Cancer. 2018;143(4):758-766.

10. Otsuka K, Imanishi Y, Tada Y, et al. Clinical outcomes and prognostic factors for salivary duct carcinoma: a multi-institutional analysis of 141 patients. Ann Surg Oncol. 2016;23(6):2038-2045.

11. Tam S, Sandulache VC, Metwalli KA, et al. Incompletely treated malignancies of the major salivary gland: toward evidence-based care. Head Neck. 2018;40(8):1630-1638.

12. Lee SH, Roh JL, Kim JS, et al. Detection of distant metastasis and prognostic prediction of recurrent salivary gland carcinomas using (18) F-FDG PET/CT. Oral Dis. 2018;24(6):940-947.

13. Roh JL, Cho KJ, Kwon GY, Choi SH, Nam SY, Kim SY. Prognostic values of pathologic findings and hypoxia markers in 21 patients with salivary duct carcinoma. J Surg Oncol. 2008;97(7):596-600.

14. Kim JY, Lee S, Cho KJ, et al. Treatment results of post-operative radiotherapy in patients with salivary duct carcinoma of the major salivary glands. Br J Radiol. 2012;85(1018):e947-e952.

15. Shinoto M, Shioyama $Y$, Nakamura K, et al. Postoperative radiotherapy in patients with salivary duct carcinoma: clinical outcomes and prognostic factors. J Radiat Res. 2013;54(5):925-930.

16. Al-Qahtani KH, Tunio MA, Bayoumi Y, Gurusamy VM, Bahamdain FA, Fatani H. Clinicopathological features and treatment outcomes of the rare, salivary duct carcinoma of parotid gland. J Otolaryngol Head Neck Surg. 2016;45(1):32.

17. Laramore GE, Krall JM, Griffin TW, et al. Neutron versus photon irradiation for unresectable salivary gland tumors: final report of an RTOG-MRC randomized clinical trial. Radiation Therapy Oncology Group. Medical Research Council. Int J Radiat Oncol Biol Phys. 1993;27(2):235-240.

18. Airoldi M, Fornari G, Pedani F, et al. Paclitaxel and carboplatin for recurrent salivary gland malignancies. Anticancer Res. 2000;20(5C): 3781-3783.

19. Johnston ML, Huang SH, Waldron JN, et al. Salivary duct carcinoma: Treatment, outcomes, and patterns of failure. Head Neck. 2016;38(Suppl 1):E820-E826.

20. Nakano K, Sato Y, Sasaki T, et al. Combination chemotherapy of carboplatin and paclitaxel for advanced/metastatic salivary gland carcinoma patients: differences in responses by different pathological diagnoses. Acta Otolaryngol. 2016;136(9):948-951.

21. Osborn V, Givi B, Lee A, et al. Characterization, treatment and outcomes of salivary ductal carcinoma using the National Cancer Database. Oral Oncol. 2017;71:41-46.

22. Nabili V, Tan JW, Bhuta S, Sercarz JA, Head CS. Salivary duct carcinoma: a clinical and histologic review with implications for trastuzumab therapy. Head Neck. 2007;29(10):907-912.

23. Schmitt NC, Kang H, Sharma A. Salivary duct carcinoma: an aggressive salivary gland malignancy with opportunities for targeted therapy. Oral Oncol. 2017;74:40-48.

24. Locati LD, Perrone F, Cortelazzi B, et al. Clinical activity of androgen deprivation therapy in patients with metastatic/relapsed androgen receptor-positive salivary gland cancers. Head Neck. 2016;38(5): 724-731.

25. Fushimi C, Tada Y, Takahashi H, et al. A prospective phase II study of combined androgen blockade in patients with androgen receptor-positive metastatic or locally advanced unresectable salivary gland carcinoma. Ann Oncol. 2018;29(4):979-984. 


\section{Publish your work in this journal}

Cancer Management and Research is an international, peer-reviewed open access journal focusing on cancer research and the optimal use of preventative and integrated treatment interventions to achieve improved outcomes, enhanced survival and quality of life for the cancer patient. The manuscript management system is completely online and includes a very quick and fair peer-review system, which is all easy to use. Visit http://www.dovepress.com/testimonials.php to read real quotes from published authors.

Submit your manuscript here: https://www.dovepress.com/cancer-management-and-research-journal 